\title{
Dabigatran and rivaroxaban, new oral anticoagulants. new approaches in dentistry
}

\author{
Gerardo Gómez-Moreno ${ }^{1}$, Antonio Aguilar-Salvatierra² ${ }^{2}$, Miguel Ángel Martín-Piedra², Javier Guardia ${ }^{3}$, José \\ Luis Calvo-Guirado ${ }^{4}$, Maribel Cabrera ${ }^{5}$, Cristina López-Gallardo ${ }^{5}$, Tania Castillo ${ }^{5}$
}

\footnotetext{
${ }^{1}$ Professor Responsible of Pharmacological Interactions in Dentistry. Faculty of Dentistry. University of Granada.

${ }^{2}$ Degree in Dentistry. Collaborator in Pharmacological Interactions in Dentistry. University of Granada.

${ }^{3}$ Doctor in Dentistry. Collaborator of Pharmacological Interactions in Dentistry. Faculty of Dentistry. University of Granada.

${ }^{4}$ Professor of Dental Clinic Integrated for Adults. Faculty of Dentistry. University of Murcia.

${ }^{5}$ Collaborator of Pharmacological Interactions in Dentistry. Faculty of Dentistry. University of Granada.
}

Correspondence:

Prof. Gerardo Gómez-Moreno.

Faculty of Dentistry, University of Granada.

Colegio Máximo s/n, Campus de Cartuja.

E-18071. Granada (España)

E-mail:ggomez@ugr.es

Received: $12 / 12 / 2009$

Accepted: 04/01/2010

\begin{abstract}
Gómez-Moreno G, Aguilar-Salvatierra A, Martín-Piedra MA, Guardia J, Calvo-Guirado JL, Cabrera M, López-Gallardo C, Castillo T. Dabigatran and rivaroxaban, new oral anticoagulants. new approaches in dentistry. $\mathrm{J}$ Clin Exp Dent. 2010;2(1):e1-5.

http://www.medicinaoral.com/odo/volumenes/v2i1/jcedv2ilp1.pdf
\end{abstract}

Article Number: $678928 \quad$ http://www.medicinaoral.com/odo/indice.htm

(C) Medicina Oral S. L. C.I.F. B 96689336 - eISSN: 1989-5488

eMail: jced@jced.es

\begin{abstract}
Treatment in patients with atrial fibrillation or venous thromboembolism in recent decades has been based almost exclusively on the use of vitamin $\mathrm{K}$ antagonists. These drugs have a narrow therapeutic index, so it is precise to repeated adjustments of doses that require analytical monitoring. For many years it has advocated the need to have more convenient new antithrombotic drugs. So is developing a new generation of antithrombotic not related to coumarin. In 2008 and 2009, two of these new anticoagulants have been registered and approved in Europe and Canada-these are dabigatran etexilate (Pradaxa ${ }^{\circledR}$ ) and rivaroxaban (Xarelto ${ }^{\circledR}$ ). Anticoagulant dabigatran is the first direct thrombin inhibitor, orally available. Specifically and reversibly inhibits thrombin, so the duration of action is predictable. The anticoagulant effect correlates well with plasma drug concentrations, which implies an effective anticoagulation with low bleeding risk without major problems of interactions with other drugs. Rivaroxaban is first oral anticoagulant inhibitor of factor Xa (FXa). It produces a predictable and reversible inhibition of FXa activity with ability to inhibit clot-bound FXa. The predictable pharmacokinetics and pharmacodynamics characteristics of dabigatran and rivaroxaban may facilitate dental management of patients who until now have been in treatment with traditional anticoagulants, given that it doesn't require routine laboratory monitoring in the vast majority of patients treated. They also present a profile of drug interactions very favourable.
\end{abstract}

Key words: Dabigatran, rivaroxaban, warfarin, heparin, anticoagulants, dentistry. 


\section{Introduction}

Oral anticoagulants are a group of drugs used to treat many cardiovascular diseases, such as in the prophylaxis of systemic embolism (whose risk is increased in patients with atrial fibrillation or have undergone orthopaedic surgery) (1). The vitamin $\mathrm{K}$ antagonists, among which are warfarin and acenocoumarol, have low therapeutic index (2) as its pharmacological management is difficult and need continuous monitoring, also have multiple interactions with other drugs and food (3). Many of the patients being treated with warfarin have an inadequate anticoagulation. Despite the great effort in creating protocols of care and appropriate use of anticoagulants available, the results in clinical practice are by no means desired (4). From this, comes the need for new anticoagulant agents that are effective, safe and easy to use. After a time when there have been little pharmacological innovations in the field of anticoagulants, the recent emergence of new oral anticoagulants has generated high expectations. Special attention should be given to direct thrombin inhibitors (dabigatran) and FXa inhibitors (rivaroxaban). These new drugs have similar effectiveness to traditional drugs, though, have fewer side effects. In addition, dental management of patients treated with the new anticoagulants may be easier because it does not require monitoring.

\section{Dabigatran}

Dabigatran etexilate (Pradaxa ${ }^{\circledR}$, Boehringer Ingelhe$\mathrm{im}$, Spain) is the first anticoagulant and reversible direct inhibitor of thrombin available orally. Specifically and reversibly inhibits thrombin, which is the key enzyme of the cascade of the coagulanlation (Fig. 1). It has a bioavailability values of $5 \%$ and once absorbed is converted in the liver to its active metabolite, dabigatran, which is mostly eliminated by the kidneys ( $80 \%$ ) (Table 1). The absorption of the Prodrug dabigatran extexilate and it's conversion to dabigratan is faster. The maximum plasma concentration was reached between 30 minutes and 2 hours after oral administration, leading to a rapid onset of effect with a half life of 12 to 17 hours (5). Despite the low oral bioavailability, it shows low variability among individuals. Studies in both healthy volunteers and patients undergoing major orthopaedic surgery have indicated that it allows fixed dosing regimens. The anticoagulant effect correlates well with plasma drug concentrations, which implies an effective anticoagulation with low bleeding risk.

\section{Dabigatran versus heparin}

The dabigatran has been evaluated as prophylactic thromboembolism venous dose of $150 \mathrm{mg}$ or $220 \mathrm{mg}$ orally after hip replacement surgery and knee, compared with enoxaparin $40 \mathrm{mg}$ orally (low molecular weight heparin) (6-8). The results of these studies were similar

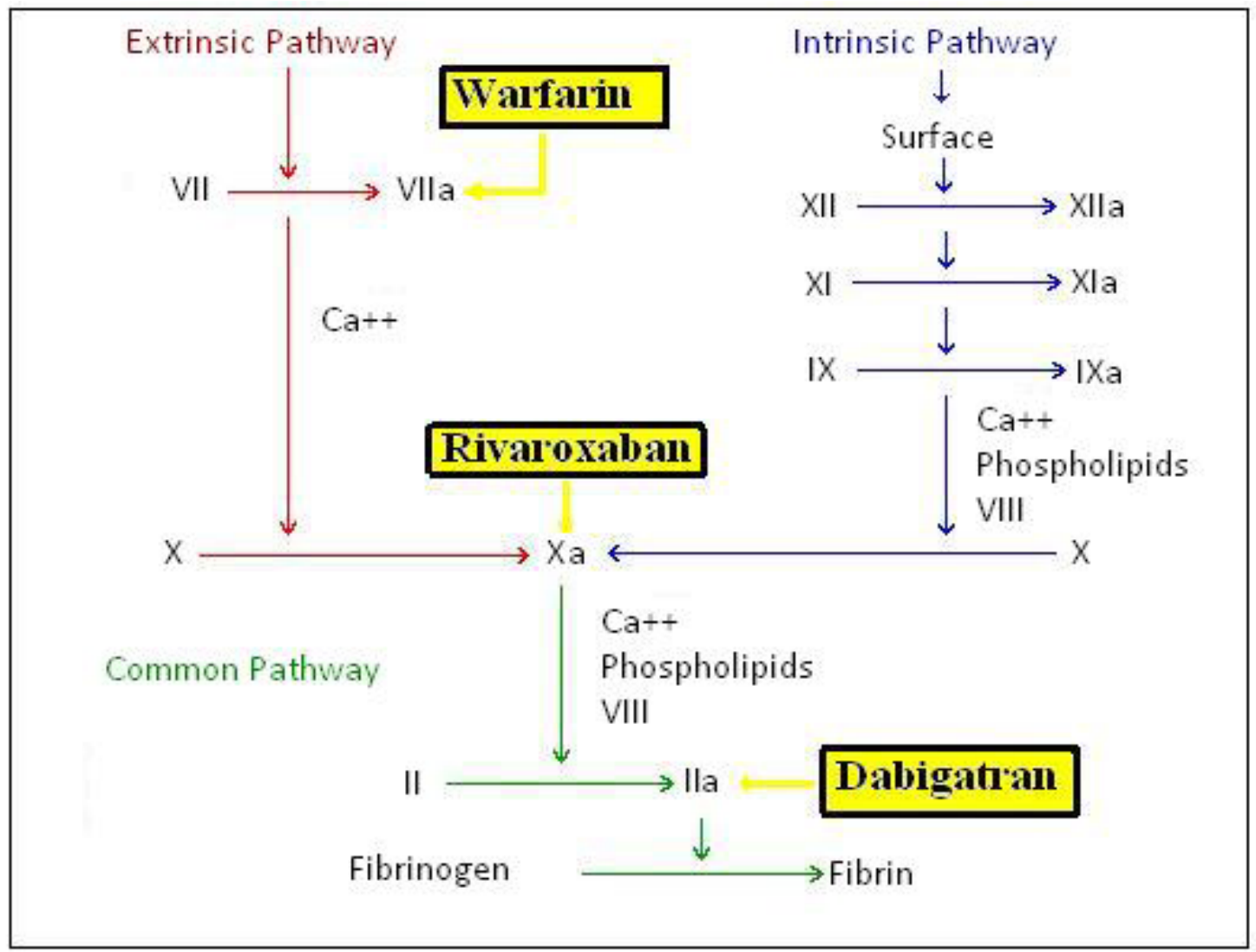

Fig.1. Mechanism of action of dabigatran, rivaroxaban and warfarin. 


\begin{tabular}{|c|c|c|c|}
\hline Type & Warfarina & Dabigatran & Rivaroxaban \\
\hline Half-life & $\begin{array}{c}\text { Vitamin K-dependent } \\
\text { factors inhibitor }\end{array}$ & $\begin{array}{c}\text { Direct thrombin in- } \\
\text { hibitor }\end{array}$ & $\begin{array}{c}\text { Direct factor Xa inh- } \\
\text { bitor }\end{array}$ \\
\hline Elimination & 2.5 days & $14-17 \mathrm{~h}$. & $5-13 \mathrm{~h}$. \\
\hline Plasmatic protein \\
binding
\end{tabular}

Table 1. Comparison of dabigatran and rivaroxaban with warfarin.

for both drugs in both surgical procedures. Furthermore, dabigatran bleeding has been associated with clinically less relevant than those produced by current patterns of low molecular weight heparins (9).

\section{Dabigatran versus warfarin}

The clinical effectiveness of dabigatran, which acts at the end of the coagulation cascade (10), seems to be very different from the effect of warfarin, which acts almost at all levels of the cascade. Recent studies that have compared warfarin and dabigatran show the latter as a substitute for warfarin in patients with atrial fibril, where the risk of thromboembolic events systemic level is increased $(5,11,12)$.Connolly et al. (2009) (12) have recently published the results of an evaluation of long-term anticoagulant therapy, comparing dabigatran, at doses of $110 \mathrm{mg}$ and $150 \mathrm{mg}$ twice daily with warfarin (classical pharmacological therapy of atrial fibrillation). The trial was conducted in 18,113 patients with atrial fibrillation for 2 years. The study results suggest that therapy with dabigatran $150 \mathrm{mg}$ twice daily is linked to lower rates of stroke and systemic embolism, although, the incidence of major bleeding events is similar when compared with the results of warfarin. There were no significant differences in mortality between patients. Despite having similar effects in terms of incidence of bleeding, treatment with dabigatran did not require continuous monitoring and was not associated with the emergence of significant adverse effects (11). In this sense, it has been suggested that patient adherence to dabigatran will be better than warfarin. The new drug is more expensive, but can greatly allow reduce costs due to the decrease in the rate of heart attacks, plus the fact of not requiring continuous monitoring. Therefore, some authors have advocated the dabigatran as a substitute in the near future of warfarin as therapy of choice for prevention of thromboembolic events and stroke in patients with atrial fibrillation, but still need more studies that reveal the possible adverse drug reactions long-term (11).

\section{Rivaroxaban}

Rivaroxaban (Xarelto ${ }^{\circledR}$, Bayer HealthCare and Johnson \& Johnson Pharmaceutical Research \& Development) is the first oral anticoagulant drug direct inhibitor of activated factor X (FXa) of clotting. It is a derivative of oxazolidone able to inhibit free factor Xa and / or attached to the prothrombinase complex. This inhibition disrupts the intrinsic and extrinsic pathways of coagulation cascade, inhibiting therefore the formation of thrombin and thrombus formation (Fig. 1). Rivaroxaban has not proved effective over the platelet action. Recent clinical trials have shown that anticoagulation can be predicted in patients with no need to adjust or monitor them. Has more than $80 \%$ bioavailability after oral administration, is metabolized by the liver and $66 \%$ is excreted in the urine (Table 1) The purpose of this new oral anticoagulant is to replace vitamin $\mathrm{K}$ antagonists in the prevention of venous thromboembolism and arterial embolism in patients with atrial fibrillation. This FXa inhibitor has been evaluated in several phase III trial studies, to study it's use in secondary thombroprophylaxis in major orthopaedic surgery, used in doses of $10 \mathrm{mg}$ orally (13-15). Rivaroxaban has been shown to be significantly more effective than treatment with enoxaparin.

\section{New approaches in dentistry}

The dental management of patients on oral anticoagulant treatment should always be painstaking, and especially to those more invasive treatments that generate or can generate bleeding (tooth extraction, oral surgery ...). The expert in oral cavity must know the therapeutic range of the patient, according to the cardiovascular disease that he presents, and based on that, assess the potential risk of bleeding prior to dental treatment. The use of oral anticoagulants is always controversial because of the low therapeutic index (2), an excessive overdose is linked to the occurrence of haemorrhage, whereas if there is insufficient plasma concentrations it increases the risk of thromboembolic phenomena occurring. This situa- 
tion requires continuous monitoring of INR (International Normal Ratio) in the case of patients treated with oral coumarin anticoagulants, among which the acenocoumarol or warfarin (16). One of the most important advantages of dabigatran and rivaroxaban, compared to classical anticoagulants, is the lack of the need for continuous monitoring in order to maintain levels of anticoagulation (12), INR values, at all times in a certain range depending on the patient's cardiovascular disease. This does not mean that the management of patients with dabigatran and rivaroxaban does not require special attention. In terms of dental treatment to be done, there will be a number of local haemostatic measures to reduce the risk of bleeding. Currently there is no reduction protocol of the dabigatran dosage or the rivaroxaban for dental use. In this sense, more scientific evidence is needed to specify the dental management to new anticoagulants, although, according to scientific evidence it seems to be much easier due to the stability of INR that is achieved with these drugs. Among other advantages of the new oral anticoagulants the highlight is that it has fewer drug interactions. Rivaroxaban does not seem to inhibit or induce any P450 enzyme. The rivaroxaban shows no relevant drug interaction with non-steroidal antiinflammatory drugs (NSAIDs). Contrary to what occurs with oral coumarin anticoagulants, which when combined lead to increased bleeding (3). It has been suggested that rivaroxaban and enoxaparin can be admistered in concomitantly form or in sequential therapy (17). Rivaroxaban prolongs the bleeding time when coadministered with aspirin or clopidogrel, although the relevance of this interaction is weak and does not affect platelet aggregation. Dabigatran, moreover, is not metabolized by the cytochrome P450 system and therefore presents a profile of drug without major problems of drug interactions. With regard to potential interactions with antimicrobial caution should be exercised only due to its pharmacokinetics, with clarithromycin and rifampicin. Regarding NSAIDs NSAIDs is not recommended to prescribe long biological half-life of elimination ( $>12$ h) in patients treated with dabigatran. Dabigatran and rivaroxaban does not require coagulation monitoring in the currently approved indications, however, there is no specific antidote (18), so that in case of overdose should maintain adequate diuresis, haemostasis or the transfusion of fresh frozen plasma (17).

\section{Conclusions}

The emergence of new oral anticoagulants, such as dabigatran and rivaroxaban, has in recent years generated great expectations in the scientific community due to: the similar clinical effectiveness of warfarin and enoxaparin over to a more predictable pharmacokinetics and pharmacodynamics and the adverse effects clinically less relevant. These new drugs appear as substitutes of coumarin anticoagulants and have the potential to change the standards of clinical practice in the prevention of deep vein thrombosis and pulmonary embolism. The use of dabigatran and rivaroxaban does not require continuous monitoring of INR. Furthermore, dabigatran and rivaroxaban, unlike warfarin or acenocoumarol, have no drug interactions with NSAIDs or with most first-choice antimicrobials used in the dental office, so it is a dental management safer and easier for the professional in the oral cavity.

\section{References}

1. Schenk JF, Stephan B, Kusma M, Groß J, Eichler H. Orthopedic patients with or without thrombophilia : Diagnostic, therapy and perioperative strategies. Orthopade. 2009 Sep 4.

2. Gómez-Moreno G, Guardia J, Cutando A, Calvo-Guirado JL. Pharmacological interactions of vasoconstrictors. Med Oral Patol Oral Cir Bucal. 2009;14:E20-7.

3. Gómez-Moreno G, Guardia J, Cutando A, Calvo-Guirado JL. Pharmacological interactions of anti-inflammatory-analgesics in odontology. Med Oral Patol Oral Cir Bucal. 2009 ;14:E81-9.

4. Warwick D, Friedman RJ, Agnelli G, Gil-Garay E, Johnson K, FitzGerald $\mathrm{G}$, et al. Insufficient duration of venous thromboembolism prophylaxis after total hip or knee replacement when compared with the time course of thromboembolic events: findings from the Global Orthopaedic Registry. J Bone Joint Surg Br. 2007;89:799-807.

5. Ezekowitz MD, Reilly PA, Nehmiz G, Simmers TA, Nagarakanti R, Parcham-Azad K, et al. Dabigatran with or without concomitant aspirin compared with warfarin alone in patients with nonvalvular atrial fibrillation (PETRO Study). Am J Cardiol. 2007;100:1419-26.

6. Eriksson BI, Dahl OE, Rosencher N, Kurth AA, van Dijk CN, Frostick SP, et al. Oral dabigatran etexilate vs. subcutaneous enoxaparin for the prevention of venous thromboembolism after total knee replacement: the RE-MODEL randomized trial. J Thromb Haemost. 2007;5:2178-85.

7. Eriksson BI, Dahl OE, Rosencher N, Kurth AA, van Dijk CN, Frostick SP, et al. Dabigatran etexilate versus enoxaparin for prevention of venous thromboembolism after total hip replacement: a randomised, double-blind, non-inferiority trial. Lancet. 2007;370:949-56.

8. Ginsberg JS, Davidson BL, Comp PC, Francis CW, Friedman RJ, Huo $\mathrm{MH}$, et al. Oral thrombin inhibitor dabigatran etexilate vs North American enoxaparin regimen for prevention of venous thromboembolism after knee arthroplasty surgery. J Arthroplasty. 2009;24:1-9.

9. Bounameaux $\mathrm{H}$. The novel anticoagulants: entering a new era. Swiss Med Wkly. 2009;139:60-4.

10. Turpie AG. New oral anticoagulants in atrial fibrillation. Eur Heart J. 2008;29:155-65.

11. Camm AJ. The RE-LY study: Randomized Evaluation of Longterm anticoagulant therapY: dabigatran vs. warfarin. Eur Heart J. 2009;30:2554-5.

12. Connolly SJ, Ezekowitz MD, Yusuf S, Eikelboom J, Oldgren J, Parekh A, et al. Dabigatran versus warfarin in patients with atrial fibrillation. N Engl J Med. 2009;361:1139-51.

13. Eriksson BI, Borris LC, Friedman RJ, Haas S, Huisman MV, Kakkar AK, et al. Rivaroxaban versus enoxaparin for thromboprophylaxis after hip arthroplasty. N Engl J Med. 2008;358:2765-75.

14. Kakkar AK, Brenner B, Dahl OE, Eriksson BI, Mouret P, Muntz J, et al. Extended duration rivaroxaban versus short-term enoxaparin for the prevention of venous thromboembolism after total hip arthroplasty: a double-blind, randomised controlled trial. Lancet. 2008;372:31-9.

15. Lassen MR, Ageno W, Borris LC, Lieberman JR, Rosencher N, Bandel TJ, et al. Rivaroxaban versus enoxaparin for thromboprophylaxis after total knee arthroplasty. N Engl J Med. 2008;358:2776-86.

16. Jiménez Y, Poveda R, Gavaldá C, Margaix M, Sarrión G. An update on the management of anticoagulated patients programmed for dental extractions and surgery. Med Oral Patol Oral Cir Bucal. 
2008;13:E176-9.

17. Ordovás Baines JP, Climent Grana E, Jover Botella A, Valero García I. Dabigatran and rivaroxaban, new oral anticoagulants for the treatment of venous thromboembolism. Farm Hosp. 2009;33:125-33.

18. Heindl B, Spannagl M . New oral anticoagulants : Consequences for perioperative coagulation diagnostics and therapy. Anaesthesist. 2009. 